\title{
A Fire Detection Algorithm Using Second Order Statistics
}

\section{J. KLOSE and R. SIEBEL}

Department of Communication Engineering, Electrical and Electronic Engineering

Duisburg University

Bismarckstr. 81, D-4100 Duisburg, Germany

\begin{abstract}
A detection algorithm is presented, based on the comparison of the short- and longterm power spectral densities of monitored input signal sequences from 'fire sensors'. The detector adapts slowly varying changes in the first and second order statistics of the monitored signals, which may occur due to environmental influences. The algorithm is as well suited for single- and multiple input detection. A recursive implementation reduces the calculation effort. Experimental results are presented, concerning the detection capability and the false alarm resistance of the detection algorithm in realistic environmental conditions.
\end{abstract}

KEYWORDS: Fire Detection, Multiple Input Detection, Second Order Statistics

\section{INTRODUCTION}

Currently installed standard fire detection systems show good detection capabilities, but further effort is required to reduce false alarms. Approximately half of the occuring false alarms are due to malfunction or unqualified handling of fire detection systems. The other half is due to non-genuine fire phenomena or unknown events [1]. At least the second half of false alarms may be reduced using improved signal detection algorithms.

Standard fire detectors are 'one input-detectors', i.e. their decision is based on the monitored signal input from one 'fire sensor'. In the simplest case the signal is directly compared with an adequately adjusted threshold value.

One approach to achieve improved discrimination between a real fire in its incipient stage and non-genuine fire phenomena are preprocessing methods with the aim, to extract distinguishing features from the observed input data. Evaluation of multiple sensor signals is very likely to achieve even better results.

A detection algorithm in this sense requires to process an input sequence or input sequences $\left\{\left\{\mathrm{x}_{1}(\mathrm{n})\right\},\left\{\mathrm{x}_{2}(\mathrm{n})\right\}, \ldots\right\}$ into a test statistic $\mathrm{D}\left[\left\{\mathrm{x}_{1}(\mathrm{n})\right\},\left\{\mathrm{x}_{2}(\mathrm{n})\right\}, \ldots\right]$, which is 
compared with a threshold value to decide, whether the fire hypothesis $\left(\mathbf{H}_{1}\right)$ or the nonfire hypothesis $\left(\mathrm{H}_{0}\right)$ is assumed.

Fire sensor signals in the incipient stage of a fire do not only show significant signal trends but also a remarkable fluctuation in comparison with the nonfire case. The decision of standard fire detectors is usually based on the first order statistics by either a direct comparison of the signal itself or some sort of expected value of the signal (smoothing or short term integration) with a threshold.

Since the detector to be presented bases its decision on second order statistics, it uses the change in the short term expected value and the fluctuation as well. Whether or not the fluctuation component is an additional distinguishing feature can be proved only, if adequate information on the statistical behaviour of stochastic fire-sensor signals under fire and non-fire conditions is available.

The Communications Department of Duisburg University, in cooperation with the SIEMENS A.G. in Munich, started in 1986 a research project supported by the Minister of Research and Technology FRG. In this project a system was developed, capable of simultaneously recording the incoming signals of different grouped sensors located in various environments with the aim of designing new detection algorithms with improved performance as well as test methods based on the recorded data [2], [3]. The data base collected with this system contains 'false alarm relevant' signal sequences for years of observation time. A performance test of the detector with this data is a realistic measure.

\section{THE DETECTION ALGORITHM}

The second order statistic of a stationary random process is described either with the autocorrelation function (ACF) $R_{X X}(\tau)$ or its fourier transform $S_{X X}(\omega)$, the power spectral density (PSD). In case of instationary processes both of these functions are dependent on the actual time $R_{X X}(t, \tau), S_{X X}(t, \omega)$.

First two stationary random processes are considered with bandlimited PSD in the range $-\omega_{g}<\omega<\omega_{g}$. The teststatistic $\mathrm{D}$ is defined as follows:

$D=\int_{-\omega_{\mathrm{g}}}^{\omega_{\mathrm{g}}}\left(\Delta S_{X X}(\omega)\right)^{2} d \omega \quad$ with $\Delta S_{X X}(\omega)=S_{X X}(\omega)-S_{X X}^{R}(\omega)$

In this formula $S_{X X}(\omega)$ represents the PSD of the monitored process and $S_{X X}^{R}(\omega)$ a reference spectrum the definition of which is given later. With $R_{X X}(k T)$ the values of the ACF $R_{X X}(\tau)$ taken at time instances $\mathrm{kT}$ corresponding to the Nyquist condition $T=\frac{\pi}{\omega_{g}}$ the PSD's in (1) can be calculated in terms of the sample-ACF:

$S_{X X}(\omega)=R_{X X}(0)+2 \sum_{k=1}^{\infty} R_{X X}(k T) \cos (k \omega T)$

$S_{X X}^{R}(\omega)=R_{X X}^{R}(0)+2 \sum_{k=1}^{\infty} R_{X X}^{R}(k T) \cos (k \omega T)$

With the following definition

$\Delta R_{X X}(k T)=R_{X X}(k T)-R_{X X}^{R}(k T) \quad k=0, \infty,(1)$ 
the teststatistic is recalculated inserting (2) and (3) in (1) and using (4)

$D=\int_{-\omega_{\mathrm{g}}}^{\omega}\left[\Delta R_{X X}(0)+2 \sum_{k=1}^{\infty} \Delta R_{X X}(k T) \cos (\omega k T)\right]^{2} d \omega$

Expansion of the square in (5) and interchanging the order of integration and summation yields

$$
\begin{aligned}
D & =\int_{-\omega_{\mathrm{g}}}^{\omega_{\mathrm{g}}}\left[\Delta R_{X X}(0)\right]^{2} d \omega+4 \sum_{k=1}^{\infty} \Delta R_{X X}(0) \Delta R_{X X}(k T) \int_{-\omega_{\mathrm{g}}}^{\omega_{\mathrm{g}}} \cos (\omega k T) d \omega+. . \\
& +4 \sum_{k=1}^{\infty} \sum_{l=1}^{\infty} \Delta R_{X X}(k T) \Delta R_{X X}(l T) \int_{-\omega_{\mathrm{g}}}^{\omega_{\mathrm{g}}} \cos (\omega k T) \cos (\omega l T) d \omega
\end{aligned}
$$

This reduces with

$$
\int_{-\omega_{\mathrm{g}}}^{\omega_{\mathrm{g}}} \cos (\omega k T) d \omega=0 \quad \text { for } k=1, \infty,(1)
$$

and the orthogonality condition

$$
\int_{-\omega_{\mathrm{g}}}^{\omega_{\mathrm{g}}} \cos (\omega k T) \cos (\omega l T) d \omega=\left\{\begin{array}{lll}
0 & \text { für } & k \neq l \\
\omega_{g} & \text { für } & k=l
\end{array}\right.
$$

to equation (9)

$D=2 \omega_{g}\left[\Delta R_{X X}(0)\right]^{2}+4 \omega_{g} \sum_{k=1}^{\infty}\left[\Delta R_{X X}(k T)\right]^{2}$

Division by $2 \omega_{\mathrm{g}}$ of this formula and use of (4) yields the normalized teststatistic $D_{n}$

$D_{n}=\left(R_{X X}(0)-R_{X X}^{R}(0)\right)^{2}+2 \sum_{k=1}^{\infty}\left(R_{X X}(k T)-R_{X X}^{R}(k T)\right)^{2}$

Unfortunately the summation index is up to infinity. The ACF can be written in terms of the autocovariance function and the expected value

$R_{X X X}(k T)=C_{X X}(k T)+E^{2}\{X\}$

If the process $X$ and the reference process $X^{R}$ are wide sense stationary and do not contain periodic signal components, the autocovariance functions decrease with increasing time shift $\mathrm{kT}$. If in this case the expected values $\mathrm{E}\{\mathrm{X}\}$ and $\left.\mathrm{E}^{\mathrm{R}}\{\mathrm{X})\right\}$ are equal, high order terms of $\Delta R_{X X}(k T)=R_{X X}(k T)-R_{X X}^{R}(k T)$ in (10) can be neglected and the upper value of the summation index can be limited to a value of $\mathrm{q}$.

Obviously the teststatistic $D_{n}$ has the following properties:

$D_{n} \geq 0$

the equality sign holding, if the reference ACF equals the actual ACF. Since $R_{X X}(0)$ is proportional to the total power of the spectrum $S_{X X}(\omega), D_{n}$ contains in addition terms corresponding to changes of the actual ACF versus the reference ACF. 
Neither in the fire nor in the non-fire case fire sensor signals can be considered as specific functions of a stationary random process. But in practice they can be viewed at least in the non-fire case as realisations of piecewise stationary processes.

With

$\underline{X}(n T)^{T}=(X(n T), X((n-1) T), \ldots, X((n-q) T))$

a sample vector of an arbitrary random process, the autocorrelation matrix (ACM) in the strong sense is defined as

$$
\begin{aligned}
& \mathbf{R}_{\mathbf{X X}}=E\left[\underline{X}(n) \underline{X}(n)^{T}\right]= \\
& =E\left[(X(n T), X((n-1) T), \ldots, X((n-q) T))^{T}((X(n T), X((n-1) T), \ldots, X((n-q) T))]\right. \\
& =\left(\begin{array}{cccc}
R_{X X}(n T, n T) & R_{X X}(n T,(n-1) T) & \ldots & R_{X X X}(n T,(n-q) T) \\
R_{X X}((n-1) T, n T) & R_{X X}((n-1) T,(n-1) T) & \ldots & R_{X X}((n-1) T,(n-q) T) \\
\cdot & \cdot & \ldots & \cdot \\
R_{X X}((n-q) T, n T) & R_{X X}((n-q) T,(n-1) T) & \ldots & R_{X X}((n-q) T,(n-q) T)
\end{array}\right)
\end{aligned}
$$

with matrix elements:

$R_{X X}((n-i) T,(n-j) T)=E[X((n-i) T) X((n-j) T)]$ for all $i, j=0, \ldots, q$

If the second order statistics are assumed not to change in a time interval $(n-q) T \leq k T \leq n T$, the matrix elements simplify to

$$
\begin{aligned}
R_{X X}((n-i) T,(n-j) T) & =R_{X X}((n-j) T,(n-i) T) \\
& =R_{X X}(n T, k T) \text { mit } k=i-j=0, \ldots, q
\end{aligned}
$$

with a corresponding ACM, valid for the time instance nT. Because it is impossible to determine the ACF from only one observed time function, time averages as estimates for the ACM-elements are used.

For simplicity of notification, the sample interval is normalized ( $T=1$ ) in the following.

With observation vectors $x(n-k)=\left(x(n-k), x(n-1-k), \ldots, x\left(n-L_{F}-k\right)\right)^{T}$, consisting of $L_{F}$ subsequently sampled observation values, a sequence $r_{X X}(n, k)$ of estimates of the $\mathrm{ACF}$-values for every time instance $\mathrm{n}$ and for each required time shift $\mathrm{k}$ is calculated according to the following rule:

$r_{X X}(n, k)=\left(x^{T}(n) \underline{x}(n-k)\right) * h_{F}(n)$

In this formula $h_{F}(n)$ denotes the pulse response of a window function with the following properties:

$h_{F}(n)=0$ for all $n<0$ and $\sum_{n=0}^{\infty} h_{F}(n)=1$

Adequately chosen window functions permit a simple recursive calculation of $r_{X X}(n, k)$. 
A rectangular window of length $L_{F}$ with pulse response:

$h_{F}(n)= \begin{cases}\frac{1}{L_{F}} & \text { for } 0 \leq n \leq L_{F}-1 \\ 0 & \text { elsewhere }\end{cases}$

yields the following recursion rule for the calculation of $r_{\mathrm{XX}}(\mathrm{n}, \mathrm{k})$ :

$r_{X X}(n, k)= \begin{cases}r_{X X}(n-1, k)+\frac{1}{L_{F}}\left[x(n) x(n-k)-x\left(n-L_{F}\right) x\left(n-\left(L_{F}-k\right)\right)\right] & \text { for } n>L_{F} \\ \frac{1}{L_{F}-k_{i}} \sum_{i=k+1}^{L_{\mathrm{F}}} x(i) x((i-k)) & \text { for } n \leq L_{F}\end{cases}$

Alternatively a normalized 'Barnwell-window' [4] with infinite impulse response

$h(n)=\left\{\begin{array}{ll}0 & \text { for } n<0 \\ (n+1)(1-\mu)^{2} \mu^{n} & \text { for } n \geq 0\end{array} \quad 0<\mu<1\right.$

yields the recursion (22).

$r_{X X}(n, k)= \begin{cases}2 \mu r_{X X}(n-1, k)-\mu^{2} r_{X X}(n-2, k)+x(n) x(n-k) & \text { for } n>L_{F} \\ \frac{1}{L_{F}-k} \sum_{i=k+1}^{L_{F}} x(i) x(i-k) & \text { for } n \leq L_{F}\end{cases}$

The effective window length $\mathrm{L}_{\text {eff }}$ of the Barnwell-window depends on the parameter $\mu$.

Instead of (10) the following time dependent teststatistic is calculated:

$d_{n}(n)=C\left\{\left(r_{X X}(n, 0)-r_{X X}^{R}(n, 0)\right)^{2}+2 \sum_{k=1}^{q}\left(r_{X X X}(n, k)-r_{X X}^{R}(n, k)\right)^{2}\right\}$

Whereby $\mathrm{C}$ is an arbitrary scaling constant.

For reasons of reduced calculational effort the summation is limited to the order of time shift $q$. Both of the terms $r^{R} \times x(n, k), r_{X X}(n, k)$ are calculated according to the same recursion (20) or (22) but different (effective) window length. A great window length yields slow adaption to time varying second-order statistics and vice versa. Consequently the reference terms $r^{R} x X(n, k)$ in (23) are calculated with a much greater window length than the corresponding terms $r_{X X}(n, k)$ with required window length in the order of the desired detection time. Finally, a threshold comparison yields the decision rule:

$d_{n}(n){\stackrel{H_{1}}{\lessgtr}}_{H_{0}} S$

If the observed process is wide sense stationary, values of the teststatistic $d_{n}(n)$ are near zero. Changes of first and second order statistics, which may occur due to slowly varying environmental conditions are adapted, as long as they are small within the reference window length, e.g. the values of the teststatistic remain near zero. Rapid changes within the reference window length will be detected.

These introductory considerations dealt with one random signal sequence $\{x(n)\}$, corresponding to the input from a single fire sensor. The extension of the detection 
algorithm for the multiple input case is possible by weighted superimposition of signals from different fire sensors according to some rules whereby it is of importance to differentiate between low pass signals with expected increasing or decreasing trends in the fire case. In technical systems the dynamic range of measured signals is always limited. If the $j$-th signal is limited in the range:

$0 \leq x_{j \min } \leq x_{j}(n) \leq x_{j \max }$

it is reasonable to assume its expected value in the non-fire case to be either near the upper or lower limit, tending in the fire case towards the corresponding opposite limit. The superimposition requires each signal with expected negative trend in the fire case to be inverted with respect to its dynamic range.

$z_{j}(n)= \begin{cases}x_{j}(n) & \text { if the expected trend in the fire case is positive } \\ x_{j \max }-x_{j}(n) & \text { if the expected trend in the fire case is negative }\end{cases}$

For $m$ different inputs $\left\{x_{j}(n), j=1 . . m\right\}$ the sequence $\{x(n)\}$ to be tested is calculated after mapping $\left\{x_{j}(n), j=1 . . m\right\}$ using (25) into $\left\{z_{j}(n), j=1 . . m\right\}$ as follows:

$x(n)=\sum_{j=1}^{m} w_{j} z_{j}(n)$

Adjustment of weights $w_{j} \geq 0$ facilitates different monitored fire characteristics to be more or less dominant in the teststatistic $\mathrm{d}_{\mathrm{n}}(\mathrm{n})$.

As an answer to the interesting question whether the fluctuation component of the signals is a distinguishing feature between the fire and nonfire case a seperate investigation was carried out based on the difference sequence:

$x^{*}(n)=x(n)-x(n-1)$

The expected value $E\left\{x^{*}(n)\right\}$ of this sequence is very close to zero even in the fire case. A slightly modified teststatistic

$d_{n}^{*}(n)=\left(\frac{r_{X^{*} X^{*}}(n, 0)-r_{X}^{R} X^{*}(n, 0)}{r_{X}^{R} X^{*}(n, 0)}\right)^{2}+2 \sum_{k=1}^{q}\left(\frac{r_{X^{*} X^{*}}(n, k)-r_{X}^{R} X^{*}(n, k)}{r_{X}^{R} X^{*}(n, 0)}\right)^{2}$

was used for this investigation. The denominator $r_{X^{*} X^{*}}^{R}(n, 0)$ in this formula represents the variance of the reference process. Normalization serves for the adaption of the teststatistic to slowly varying changes of the signal variance due to environmental conditions.

\section{EXPRIMENTAL RESULTS}

The detection algorithms based on $d_{n}(n), d_{n}^{*}(n)$ were tested with recorded data for non-fire conditions in different environments as well as for fire conditions with EN 54 testfires [5]. Three primary fire characteristics from different grouped sensors (temperature and two smoke measurements, backscattering light- and an ionisation chamber), of simultanously recorded data with sample rate $1 / \mathrm{sec}$ were at disposal.

The multiple input performance of the teststatistics is presented. 


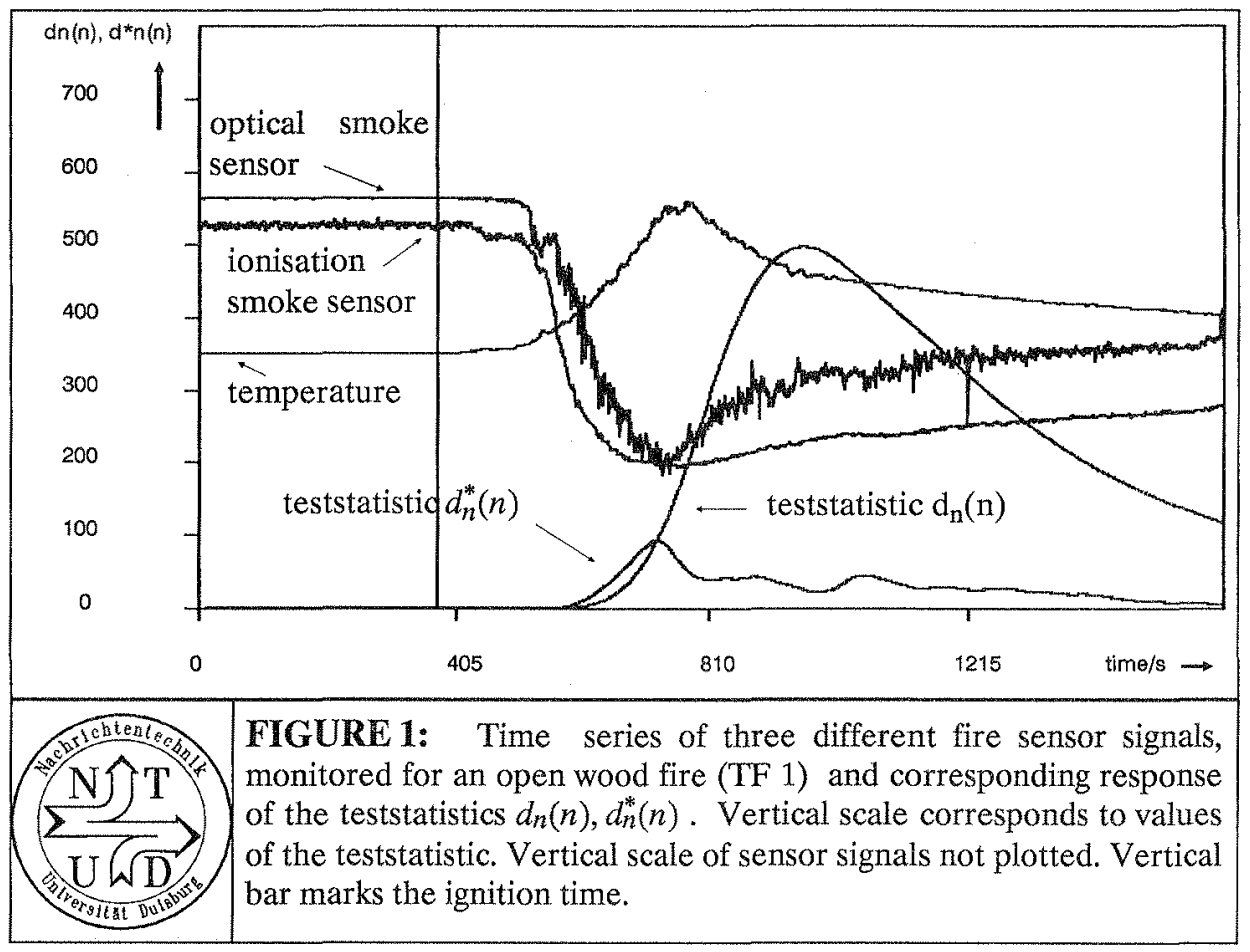

The results for all of the investigations presented in this chapter were carried out with the following parameter set:

Weights $w_{i}$ used for the superimposition of signals according to (27): $w_{1}=1$ (temperature); weight $w_{2}=1$ (optical scattering light smoke sensor); weight $\mathrm{w}_{3}=1$ (ionisation chamber smoke sensor)

Effective window lengths: $L_{\text {eff }}^{R}=5000$ ( normalized reference Barnwell window), $L_{\text {eff }}=500$ (normalized main Barnwell window)

Fig. 1 shows three signals for testfire TF 1 and the response of the teststatistics $d_{n}(n), d_{n}^{*}(n)$ plotted in vertical direction on an arbitrary scale against time (n) as an example for the response behaviour of the teststatistic. The vertical bar marks the time of ignition. With a delay corresponding approximately to half of the main window length, the values of the teststatistics increase rapidly. The adjustment of a threshold value for each teststatistic $d_{n}(n), d_{n}^{*}(n)$ according to (24) would indicate the corresponding alarm times. But adequate threshold setting requires information of the teststatistic behaviour in various fire- and non-fire environments which is given in the following.

Both teststatistics were calculated for a total of 45 testfire runs to achieve a profound test for the detection capability. Signals from six groups of sensors in different locations at the ceiling of the fire laboratory $(4 \mathrm{~m}$ height, $1 . .5 \mathrm{~m}$ horizontal distance from the 
TABME 1. Lowest and highest response maximun of the teststatistic for different EN 54 testrires

\begin{tabular}{|c|c|c|c|c|c|c|}
\hline \multirow{2}{*}{$\begin{array}{l}\text { Test } \\
\text { fire }\end{array}$} & \multicolumn{2}{|c|}{ Fire characteristics } & $\begin{array}{l}\text { No. of } \\
\text { testfire } \\
\text { runs }\end{array}$ & \begin{tabular}{l} 
lowest $\begin{array}{l}\text { Maximum } \\
\text { of }\end{array}$ \\
\cline { 4 - 8 }
\end{tabular} & & \multicolumn{2}{l|}{$\begin{array}{l}\text { highest } \\
\text { Maximum } \\
\text { of }\end{array}$} \\
\hline TF 1 & $\begin{array}{l}\text { Open wood fire: light smoke and } \\
\text { temperature development }\end{array}$ & 12 & 117 & 13 & 542 & 116 \\
\hline TF 2 & $\begin{array}{l}\text { Wood smoldering fire: no increase of } \\
\text { temperature; light smoke development }\end{array}$ & 9 & 82 & 9 & 300 & 253 \\
\hline TF 3 & $\begin{array}{l}\text { Cotton smoldering fire: no increase of } \\
\text { temperature; rapid smoke development }\end{array}$ & 5 & 152 & 6 & 342 & 148 \\
\hline TF 4 & $\begin{array}{l}\text { Polyurethane fire: weak temperature and } \\
\text { dark smoke development; }\end{array}$ & 6 & 69 & 13 & 378 & 171 \\
\hline TF 5 & $\begin{array}{l}\text { N-Heptane fire without Toluol additive: } \\
\text { slow dark smoke and rapid temperature } \\
\text { development }\end{array}$ & 5 & 164 & 10 & 598 & 187 \\
\hline TF 6 & $\begin{array}{l}\text { Pure methylated spirit fire: no smoke but } \\
\text { rapid temperature development }\end{array}$ & 8 & 20 & 3 & 88 & 36 \\
\hline
\end{tabular}

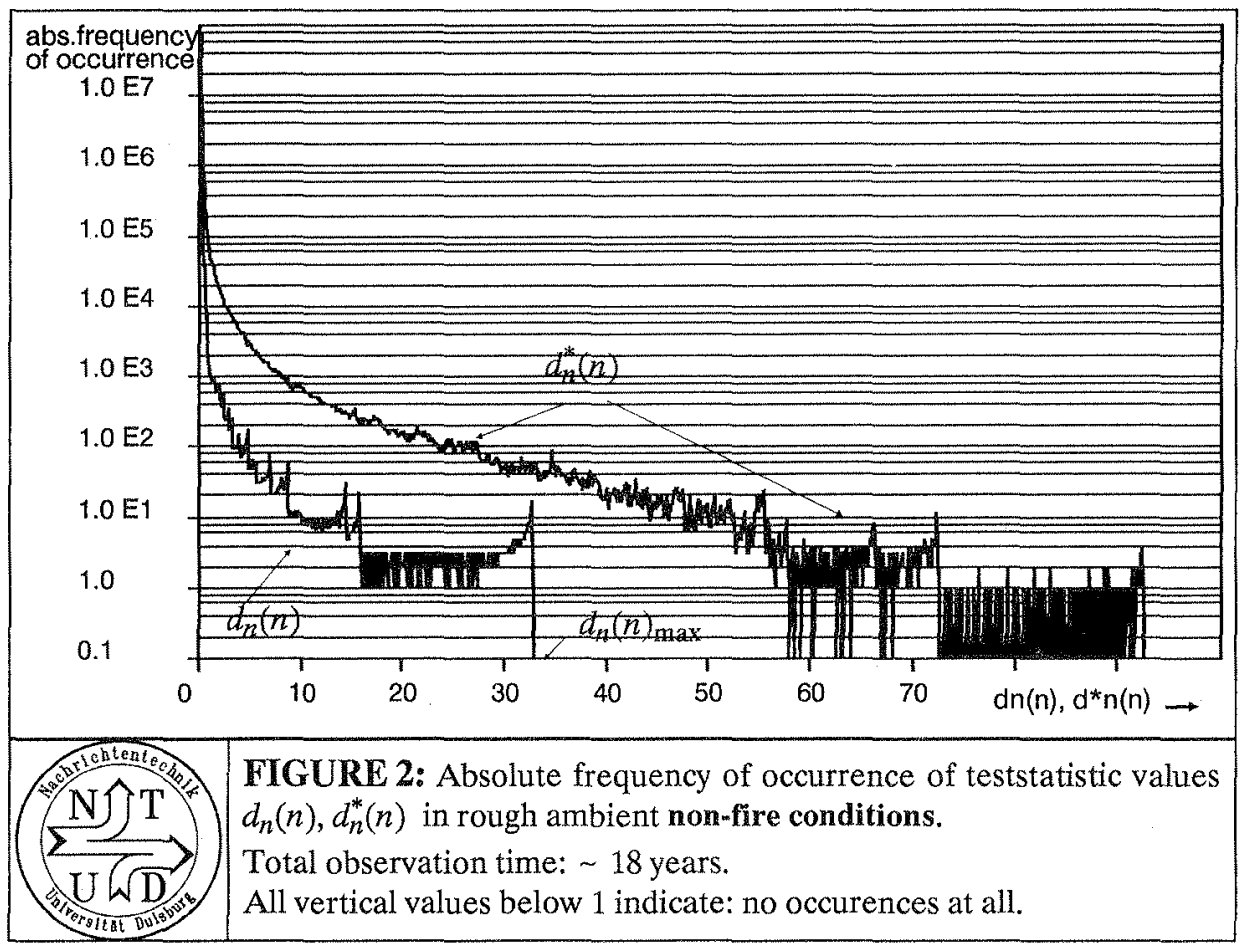


center of the fire, the location of two groups chosen arbitrarily unfavourable), were monitored for each of the testfire runs.

Table 1 shows the lowest and highest response maxima of the teststatistics $d_{n}(n), d_{n}^{*}(n)$, ordered for six different types of EN 54 testfires, the lowest (highest) maximum indicating the worst (best) result of all ( $6 \mathrm{x}$ (No. of testfire runs)) calculated teststatistics in each line.

Fig. 2 shows the absolute frequency of the occurrence of $d_{n}(n), d_{n}^{*}(n)$ values for the non-fire case. Fixing of a threshold on the horizontal axis enables the count out of the number of threshold crossings (alarm condition). Recorded sensor signals in six different locations of each a steel factory (production line, cable duct, oil pressure supply room etc.), the supply rooms of a hospital (canteen kitchen, laundry, desinfection room, power- and air conditioning supply etc.) and an underground car park were used for this investigation. All of these installation locations are to be considered as extremely false alarm sensitive due to abnormal dust-, steam-, smoke- and temperaturedevelopment. The total observation time is approximately 18 years.

\begin{tabular}{|c|c|c|c|c|c|c|c|c|}
\hline Table $2 *$ Perfor & liresh & son & mere & novin & arerage & $t=20$ & 1, & tors \\
\hline Missing & alarn & ns of & thresho & detect & ars for & ifferent & est-fire & \\
\hline Testfire & $\begin{array}{l}\text { No. } \\
\text { of } \\
\text { Fires }\end{array}$ & $\begin{array}{l}\text { No. } \\
\text { of } \\
\text { Sen- }\end{array}$ & $\begin{array}{r}\text { Ionisati } \\
\text { det } \\
\text { Thresh }\end{array}$ & $\begin{array}{l}\text { n smoke } \\
\text { ctor } \\
\text { d: } 2000\end{array}$ & $\begin{array}{r}\text { Optice } \\
\text { det } \\
\text { Thresh }\end{array}$ & $\begin{array}{l}\text { smoke } \\
\text { ctor } \\
\text { ld: } 2000\end{array}$ & $\begin{array}{r}\text { Temp } \\
\text { dete } \\
\text { Thresho }\end{array}$ & $\begin{array}{l}\text { erature } \\
\text { ctor } \\
\text { ld: } 1500\end{array}$ \\
\hline & & sors & $\begin{array}{l}\text { missed } \\
\text { alarms }\end{array}$ & $\begin{array}{l}\text { detec- } \\
\text { tion rate }\end{array}$ & missed & $\begin{array}{c}\text { detec- } \\
\text { tion rate }\end{array}$ & $\begin{array}{l}\text { missed } \\
\text { alarms }\end{array}$ & $\begin{array}{c}\text { detec- } \\
\text { tion rate }\end{array}$ \\
\hline TF1 wood fire & 14 & 6 & 0 & $100 \%$ & 2 & $97.6 \%$ & 2 & $97.6 \%$ \\
\hline TF2 smoldering & 9 & 6 & 4 & $92.5 \%$ & 0 & $100 \%$ & all 54 & $0 \%$ \\
\hline TF3 smoldering & 5 & 6 & $\theta$ & $100 \%$ & 0 & $100 \%$ & all 30 & $0 \%$ \\
\hline TF4 polyurethane & 6 & 6 & 0 & $100 \%$ & 0 & $100 \%$ & 25 & $30.5 \%$ \\
\hline TF5 N-Heptane & 7 & 6 & 0 & $100 \%$ & 0 & $100 \% \%$ & 0 & $100 \%$ \\
\hline TF6 methyl,spirit & 8 & 6 & all 48 & $0 \%$ & all 48 & $0 \%$ & 0 & $100 \%$ \\
\hline & & 0 & & 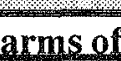 & - & $1 \mathrm{~d}$ & & \\
\hline $\begin{array}{r}\text { Total obser } \\
18 \mathrm{y} \\
\end{array}$ & ion 1 & & & & & & & \\
\hline
\end{tabular}

Table 2 shows the performance of 3 one-input threshold detetectors calculated on the same data base. We are aware of comparing one-input with multiple-input detectors to be unfair. But the results in this table show as an example for a simple detector that there are missing alarms and false alarms in each column, which indicates that there is no threshold setting at all for this detector type to achieve $100 \%$ detection rate without false alarms. 


\section{DISCUSSION}

If the highest teststatistic values in the non-fire case are smaller than the lowest maximum values (worst case) in the fire case, an adjustment of thresholds is possible to detect all of the test fires without any false alarm. This is true for any setting of the treshold value in the range from $32<\mathrm{S}<69$ for $d_{n}(n)$, if only the testfires TF1 .. TF5 are concerned. The relatively poor response of both teststatistics $d_{n}(n), d_{n}^{*}(n)$ against testfire TF 6 is due to the fact, that there is no smoke development, which is very unlikely to occur in genuine fires. But with an adequate setting of weights $w_{\mathbf{i}}$ the detector can be adjusted to meet specific risks and thereby it would even detect a fire like TF 6.

The presentation in Fig. 2 does not reflect the time instances at which the counted values occured. Further investigations showed all values between 15 and the maximum value 32 of $d_{\mathfrak{n}}(n)$ to belong to only one continuously within 3 minutes recorded situation, where smoke and temperature developement in the canteen kitchen was very similar to a real fire situation. Since for all other situations the teststatistic produced no value greater than 12 , a threshold value setting in the range $12<\mathrm{S}<20$ would be reasonable as well. In this case all testfires inclusive TF6 would have been detected and 1 additional alarm in the situation mentioned above, where unfortunately it is not known what really happend and consequently there is no hint, whether it has to be qualified as a false alarm or not.

The comparison of maximum values in the fire case with mainly occuring values in the non-fire case for the teststatistic $d_{n}^{*}(n)$ indicates the fluctuation component of the signals to be a weak distinguishing feature, because all of the highest maximum teststatistc values for testfires TF1.TF5 are considerably higher than those in non-fire situations but overlapping of some values under either hypothesis $\mathrm{H}_{0}, \mathrm{H}_{1}$ would have led to false alarms or missing of alarms for any setting of a threshold value, if a decision is solely based on $d_{n}^{*}(n)$. For this reason the use of $d_{n}^{*}(n)$ is not recommend.

Reversely the results achieved with $d_{n}^{*}(n)$ justify the original design rule (1) for the teststatistic $d_{n}(n)$, which utilizes not only the low pass component of the input signal(s) but in addition high frequency fluctuation components.

\section{CONCLUSION}

The detection algorithm based on the teststatistic $d_{n}(n)$ shows good detection capabilities for all types of testfires TF 1 .. TF 5 and excellent false alarm rejection, taking into consideration the worst case environmental conditions for investigations in both cases.

Its main advantages are applicability for one-input or multiple-input detection and its adaptive features in the sense, that slowly varying changes of first and second order statistics do not change the detection capability, as long as they are small within the reference window length. An additional attractive feature for multiple input applications is the possibility to adjust the weights $w_{j}(27)$ in such a way that with respect to the risk to be met different fire characteristics may dominate. 


\section{REFERENCES}

[1] Kunz, Fritz; Automatische Brandmeldeanlagen - ihre Wirksamkeit; Falschalarme und Maßnahmen zu ihrer Reduzierung. pp.11-24, *** see below

[2] Post, Ottfried; Ein Meßwerterfassungs-und Registriersystem für Brandkenngrößen, pp. 339-362, ***ssee below

[3] Klose, J.; Analyse und Simulation von Brandkenngrößenverläufen, pp. 363-390 **** see below

[4] Barnwell, T.P.; Recursive Windowing for Generating Autocorrelation Coefficients for LPC Analysis, IEEE Transactions on Acoustics Speech and Signal Processing, Vol. ASSP-29, No. , Oct. 1981, pp. 1062-1066

[5] Comite Europeen de Normalisation CEN; Europäische Norm EN 54; Bestandteile automatischer Brandmeldeanlagen Teil 9 .

*** all published in PROCEEDINGS zur 9. Internationalen Konferenz über automatische Brandentdeckung AUBE'89; Universität Duisburg (Herausgeber H. Luck) 1989; isbn 3-925714-24-3 
